\title{
ON SYMMETRY OF BIRKHOFF-JAMES ORTHOGONALITY OF LINEAR OPERATORS ON FINITE-DIMENSIONAL REAL BANACH SPACES
}

\author{
Debmalya Sain, Puja Ghosh and Kallol Paul
}

\begin{abstract}
We characterize left symmetric linear operators on a finite dimensional strictly convex and smooth real normed linear space $\mathbb{X}$, which answers a question raised recently by one of the authors in [7] [D. Sain, Birkhoff-James orthogonality of linear operators on finite dimensional Banach spaces, J. Math. Anal. Appl. 447 (2017) 860-866 ]. We prove that $T \in B(\mathbb{X})$ is left symmetric if and only if $T$ is the zero operator. If $\mathbb{X}$ is two-dimensional then the same characterization can be obtained without the smoothness assumption. We also explore the properties of right symmetric linear operators defined on a finite dimensional real Banach space. In particular, we prove that smooth linear operators on a finite-dimensional strictly convex and smooth real Banach space can not be right symmetric.
\end{abstract}

Mathematics subject classification (2010): Primary 47L05, secondary 46B20.

Keywords and phrases: Birkhoff-James orthogonality, symmetry of orthogonality, bounded linear operators, finite dimensional Banach spaces.

\section{REFERENCES}

[1] G. Birkhoff, Orthogonality in linear metric spaces, Duke Mathematical Journal, 1 (1935) 169-172.

[2] P. Ghosh, D. SAIN AND K. PAul, Orthogonality of bounded linear operators, Linear Algebra and its Applications, 500 (2016) 43-51.

[3] R. C. JAMES, Inner product in normed linear spaces, Bulletin of the American Mathematical Society 53, 559-566 (1947a).

[4] R. C. JamES, Orthogonality and linear functionals in normed linear spaces, Transactions of the American Mathematical Society 61, 265-292 (1947b) 69 (1958) 90-104.

[5] K. PAUl, D. SAIN AND K. JHA, On strong orthogonality and strictly convex normed linear spaces, Journal of Inequalities and Applications 2013, 2013:242.

[6] K. Paul, D. SAin, P. Ghosh, Birkhoff-James orthogonality and smoothness of bounded linear operators, Linear Algebra and its Applications, 516 (2016) 551-563.

[7] D. S AIN, Birkhoff-James orthogonality of linear operators on finite dimensional Banach spaces, Journal of Mathematical Analysis and Applications, 447 (2017) 860-866.

[8] D. SAIN, On the norm attainment set of a bounded linear operator, arXiv:1608.00755 [math.FA].

[9] D. SAIN AND K. PAUL, Operator norm attainment and inner product spaces, Linear Algebra and its Applications, 439 (2013) 2448-2452.

[10] D. SAIN, K. PAUl AND S. HAIT, Operator norm attainment and Birkhoff-James orthogonality, Linear Algebra and its Applications, 476 (2015) 85-97.

[11] A. TURNSEK, On operators preserving James' orthogonality, Linear Algebra and its Applications, 407 (2005), 189-195. 